\title{
Pulsar Period and Magnetic Field Evolution
}

\author{
F. Camilo \\ The University of Manchester, NRAL, Jodrell Bank, Macclesfield \\ Cheshire SK119DL, UK. e-mail: fernando@jb.man.ac.uk.
}

\begin{abstract}
One of the important unsolved problems in pulsar astrophysics concerns the formation and evolution of their magnetic fields. We summarize measurements of braking indices and their implications for the spin and magnetic field evolution of young pulsars. An analysis of the period-period derivative diagram suggests one or more of the following: (a) a substantial population of young pulsars remains undiscovered; (b) a large fraction of all slow pulsars may be recycled; (c) magnetic fields in isolated pulsars decay by a factor of a few in the first few million years. We also note that the observed $P-\dot{P}$ distribution of millisecond pulsars may be shaped by luminosity evolution.
\end{abstract}

\section{Introduction}

Five hundred and fifty radio pulsars with measured periods and period derivatives are plotted in the " $P-\dot{P}$ diagram" of Figure 1 , useful for describing the spin evolution of pulsars. Pulsars are thought to be born near the top left hand corner of the diagram, with relatively short periods and high magnetic fields, moving rapidly into the region of the diagram where most pulsars are found. While the association of pulsars with their respective supernova remnants, SNRs, is in many cases uncertain (see Kaspi, this volume), the most plausible associations are indicated in Figure 1. We will not discuss pulsars located in globular clusters since their evolution is probably significantly different, and also the inferred values of magnetic fields are often contaminated by cluster accelerations; see Kulkarni \& Anderson (1996) for a review. With a few assumptions about the pulsar spindown mechanism (§2.), the surface magnetic dipole field strength of a pulsar is inferred from its values of $P$ and $\dot{P}: B=3.2 \times 10^{19}(P \dot{P})^{1 / 2} \mathrm{G}$ (Manchester \& Taylor 1977; we plot a line of constant $B$ in Fig. 2a).

The spin evolution of a pulsar depends crucially on $B$ and its evolution. Soon after the discovery of pulsars, statistical studies of the population suggested that magnetic fields probably decayed exponentially on time scales of a few million years (Gunn \& Ostriker 1970). Despite this conclusion, the direct observational evidence in its support was not overwhelming, resting on a comparison of "kinetic" and "characteristic" ages (Lyne, Anderson, \& Salter 1982). Such studies generally found that the kinetic ages of pulsars $(z / \dot{z}$ where $z$ is a pulsar's distance off the Galactic plane and $\dot{z}$ its measured rate of change), were smaller than their characteristic ages (a measure of the spindown time from a much faster rotation rate, assuming constant $B ; \S 2$.). If $B$ was once larger the 


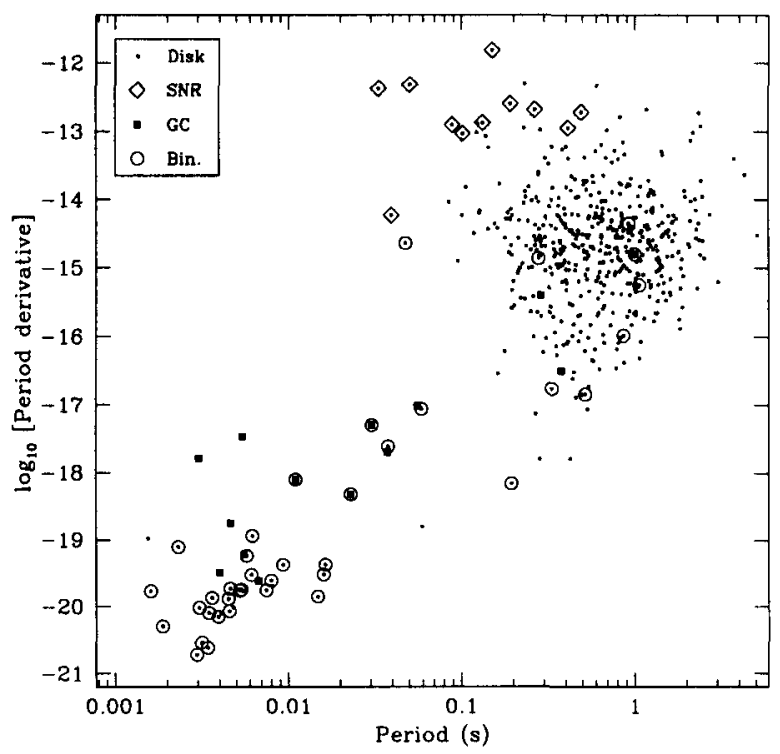

Figure 1. Measured periods and period derivatives for 550 pulsars. Special symbols denote pulsars possibly associated with supernova remnants, located in globular clusters, and in binary systems.

present characteristic age is an overestimate of the true age, and magnetic field decay therefore provided a natural explanation for the observations. Recently however, statistical studies have reached conflicting conclusions regarding the need for magnetic field decay (e.g., Narayan \& Ostriker 1990; Bhattacharya et al. 1992), and much observational evidence no longer appears to require field decay (Harrison, Lyne, \& Anderson 1993; see also Lyne \& Lorimer 1994). Furthermore, spontaneous exponential decay of magnetic fields on time scales of millions of years is incompatible with residual fields of $\gtrsim 10^{10} \mathrm{G}$ in systems many hundreds of million years old (Kulkarni 1986).

\section{Braking Indices}

The rotation rate of a pulsar, $\nu$, is usually assumed to decrease according to a power law of the form

$$
\dot{\nu}=-K \nu^{n},
$$

where $n$ is the braking index. For rotational energy loss from a constant dipolar magnetic field $n=3$, and $K \propto M^{2} / I$ where $M$ is the magnetic moment of the star and $I$ its moment of inertia (Manchester \& Taylor 1977 and references therein). Assuming constant $K$, differentiation of eq. (1) gives $n=\nu \ddot{\nu} /(\dot{\nu})^{2}$, and $n$ can be obtained from a measurement of the secular rate of change of the spindown rate, $\ddot{\nu}$. Because of the effects of glitches and timing noise (see Lyne, this volume), this has been done for only 4 pulsars, with results summarized in 
Table 1. Observed braking indices for four pulsars.

\begin{tabular}{llll}
\hline \hline PSR & \multicolumn{1}{c}{$n$} & \multicolumn{1}{c}{$\tau_{c}(\mathrm{yr})$} & \multicolumn{1}{c}{ References } \\
\hline B0531+21 & 2.51 & 1300 & Lyne, Pritchard, \& Smith 1993 \\
B1509-58 & 2.837 & 1500 & Kaspi et al. 1994 \\
B0540-69 & 2.24 & 1700 & Boyd et al. 1995 \\
B0833-45 & 1.4 & 11,000 & Lyne et al. 1996 \\
\hline
\end{tabular}

Table 1. Integration of eq. (1) with constant $K$ yields the age of a pulsar:

$$
\tau=-\frac{\nu}{(n-1) \dot{\nu}}\left[1-\left(\frac{\nu}{\nu_{i}}\right)^{n-1}\right] .
$$

For $n=3$ and $P_{i} \ll P$, where $P_{i}=1 / \nu_{i}$ is the initial spin period of the pulsar, eq. (2) reduces to $\tau_{c}=P /(2 \dot{P})$, the characteristic age of a pulsar, which we include in Table 1.

As none of the 4 measured values of braking index is consistent with $n=3$, expected from the simplest spindown models, we briefly consider some possible reasons for the discrepancy. If $K$ in eq. (1) is indeed constant, $n<3$ may be caused by an outflow of charged particles that remove mechanical angular momentum from the star, or by magnetospheric currents that distort the otherwise dipolar magnetic field (e.g., Blandford \& Romani 1988). Alternatively the correct spindown law may be of the form $\dot{\nu}=-K(t) \nu^{n}$. In this case the observed braking index $n_{\mathrm{obs}}=\nu \ddot{\nu} /(\dot{\nu})^{2}$ is not equal to the braking index $n$ of the spindown law; instead (Blandford \& Romani 1988)

$$
n-n_{\mathrm{obs}}=-\frac{\nu}{\dot{\nu}} \frac{\dot{K}(t)}{K(t)}=2 \tau_{\mathrm{c}} \frac{\dot{K}(t)}{K(t)} .
$$

With an assumed value for $n$ (e.g., 3), eq. (3) gives the time scale for the variation of $K$. From eq. (3) and the values of $n_{\text {obs }}$ in Table $1, \dot{K}>0$ for all 4 pulsars if $n \simeq$ 3 . This increase in $K \propto M^{2} / I$ may be due to an increasing magnetic moment or decreasing effective moment of inertia on a time scale of a few thousand years. Determination of $\dddot{\nu}$ would provide a check on the functional form of $K(t)$ but the measurements available to date are not very restrictive (see Kaspi et al. 1994).

A few important conclusions follow from the above summary. No measured braking indices are consistent with the typically assumed $n=3$. The few measured values are for very young pulsars and it is unclear what, if any, evolution might take place and, in particular, what $n$ may be for the bulk of "middleaged" pulsars, and also for millisecond pulsars. The four tracks in Figure 2a show the evolution over the next $10,000 \mathrm{yr}$ for the pulsars with measured values of $n$, assuming constant $n$. If this assumption is valid, all 4 pulsars will end up within the circle labeled " 2 " after a few tens of thousands of years. We now examine Figure 2a is more detail in the context of magnetic field evolution. 

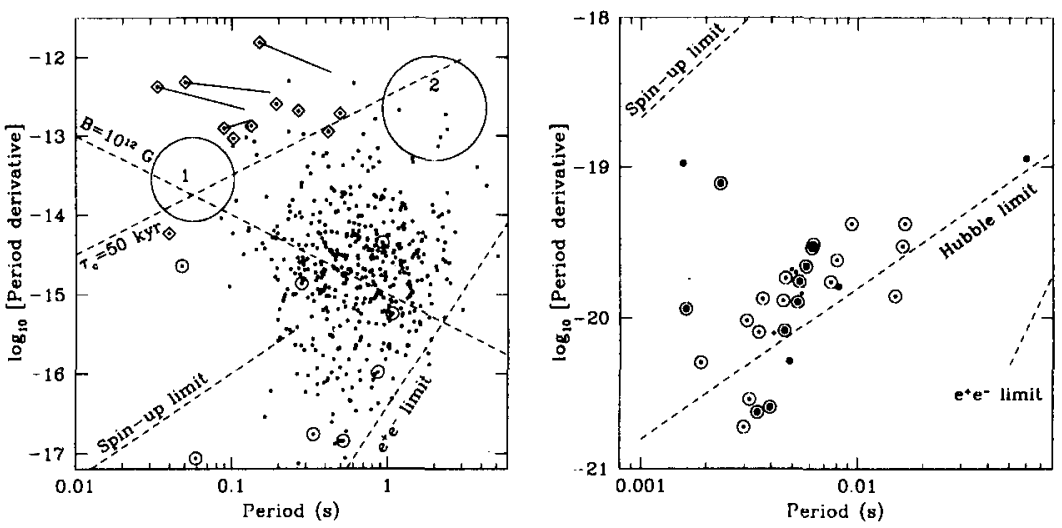

Figure 2. Details from Figure 1, with pulsars in globular clusters excluded. (a) Top right hand corner from Fig. 1. A line of constant age and one of constant magnetic field strength are drawn. See text for explanation of other lines and discussion. (b) Lower left hand corner from Fig. 1. Where a pulsar proper motion is available, $\dot{P}$ has been corrected from the measured value, as indicated by a dot of larger size.

\section{Magnetic Field Evolution}

There are very few pulsars known in region 2 of the $P-\dot{P}$ diagram in Figure 2a. As all pulsars with measured $n$ will end up there if $n$ remains constant, two possible explanations arise: either pulsars with $P \gtrsim 1$ s and $\dot{P} \gtrsim 10^{-13.5}$ suffer a somewhat drastic luminosity evolution, or $n$ is not constant (i.e., equivalently in the present context, $B$ decays and pulsars turn downward in the $P-\dot{P}$ diagram before reaching region 2 ).

The bulk of observed pulsars are located in the "island" centered on $P \sim$ $0.5 \mathrm{~s}$ and $\dot{P} \sim 10^{-14.5}$ (Fig. $2 \mathrm{a}$ ). If $B$ does not decay, there are no known progenitors for the vast majority of these pulsars: even with $n \simeq 3$, most of the young pulsars in Figure 2a, evolving along lines of constant $B$, will reach $P \sim 0.5$ s with $10^{-13.5} \lesssim \dot{P} \lesssim 10^{-12.5}$, much larger than those of most observed pulsars. It is possible that many young pulsars remain undiscovered, i.e., that the distribution of pulsars in Figure $2 \mathrm{a}$ is beset by observational selection effects such that enough pulsars actually exist near region 1 to evolve with constant $B \sim 10^{12.5} \mathrm{G}$ into the island of observed middle-aged pulsars. This missing sample of young pulsars would have $P \lesssim 50 \mathrm{~ms}$; furthermore, since they would be located predominantly along the Galactic plane and in SNRs, in regions with high background temperatures, they would be selected against in most surveys. A careful treatment of the degree to which the sample of pulsars observed in SNRs is affected by selection effects is difficult, but may answer this important question.

If one regards the observed sample of pulsars in SNRs as representing the intrinsic distribution reasonably well, one of two conclusions appears likely. There may be some decay of magnetic field in the pulsar population - not exponen- 
tially, on a time scale of a few million years, which recent population synthesis studies have rejected (Bhattacharya et al. 1992, Wakatsuki et al. 1992), but by a small factor in the first million years or so, with very slow subsequent decay. This can occur if the field is initially confined to the outer crust of the neutron star, as the star initially cools and the conductivity increases (Urpin \&. Muslimov 1992). Alternatively, or in addition, a sizable fraction of the observed isolated pulsars may be recycled, i.e., they were once part of binary systems and their magnetic fields were reduced, perhaps due to Roche-lobe overflow accretion (Romani 1990, Geppert \& Urpin 1994), or during the wind-accretion spindown phase to long periods, if the magnetic field resides in the core of the neutron star (Jahan Miri \& Bhattacharya 1994). Pulsars spun up by mass accretion at constant $B$ are thought to lie below the spin-up limit of Figure 2 (e.g., Bhattacharya \& van den Heuvel 1991), and it has been suggested that many isolated slow pulsars may indeed be recycled (Deshpande, Ramachandran, \& Srinivasan 1995). Additionally it is possible that pulsars presently observed above the spin-up limit were once spun up in binary systems: e.g., if the accreted matter temporarily screens the field, spin-up to short periods is possible; eventual diffusion of the field advected below the surface layers of the star may push the observed pulsar above the spin-up limit (Young \& Chanmugam 1995).

\section{Binary and Millisecond Pulsars and Death Lines}

After some $\sim 10^{7} \mathrm{yr}$ as luminous radio sources, as the magnetospheric processes responsible for emission cease to work efficiently, isolated pulsars cross the pairproduction threshold indicated in Figure 2 (see Chen \& Ruderman 1993). During this time, as suggested in the previous section, the magnetic field strength of these pulsars probably decreases by a factor of a few $(\$ 10)$, but no more.

In Figure $2 \mathrm{~b}$ we display the millisecond pulsar region of the $P-\dot{P}$ diagram. Where possible, the measured values of $\dot{P}$ have been corrected after accounting for the time derivative of the Doppler shift, a significant effect for many nearby millisecond pulsars (Camilo, Thorsett, \& Kulkarni 1994). The inferred magnetic fields lie in the range $8 \times 10^{7}-3 \times 10^{9} \mathrm{G}$, and some $80 \%$ of observed millisecond pulsars are found in binary systems, contrasting with the much higher $B$ and $\sim 1 \%$ binary incidence among slow pulsars. This correlation strongly suggests that the very low $B$ of millisecond pulsars is somehow linked with their evolution in binary systems. Two broad classes of models relate evolution in binary systems and field reduction: those in which the field reduction is a direct consequence of the accreted matter, e.g., due to a dragging and advection of field lines into the crust (Romani 1990), or to enhanced ohmic decay and thermomagnetic drift caused by compression and heating of the crust (Geppert \& Urpin 1994, Urpin \& Geppert 1995); and those in which it is related to the spin evolution of the neutron star. One idea involves the expulsion of the core flux into the crust, where it decays by ohmic dissipation, due to interpinning of magnetic fluxoids and angular momentum-carrying vortices, as the star spins down to very long periods in the wind-accretion phase of binary evolution. Calculations of the coupled spin-binary evolution of low mass binary systems in this framework yield testable predictions for $B$ as a function of binary parameters (Jahan Miri \& Bhattacharya 1994), which can be compared with the many recently discov- 


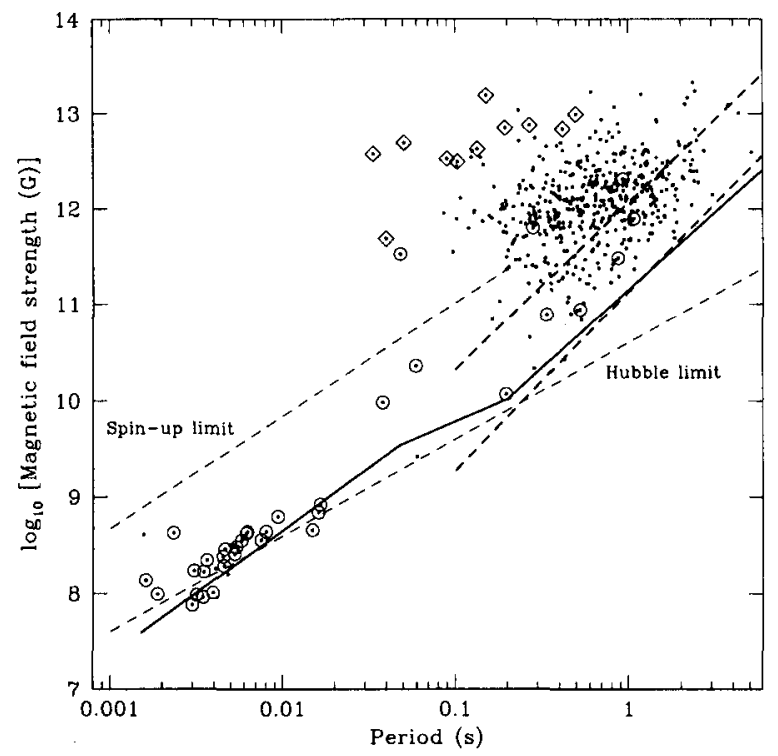

Figure 3. The same data as in Figure 2 but presented with $B$ instead of $\dot{P}$. The spin-up and Hubble limits are as for Fig. 2. The two nearly parallel dashed lines at $P>0.1$ s delimit the "death valley," within which radio emission is expected to cease (Chen \& Ruderman 1993). The solid line is the death line calculated by Björnsson (1996).

ered systems (Fig. 2b). In addition the lowest possible $B$ obtainable via this mechanism is $\sim 10^{8} \mathrm{G}$, close to observed values.

With the exception of the two nearest the spin-up limit, the pulsars in Figure $2 \mathrm{~b}$ have characteristic ages between 2 and $25 \times 10^{9} \mathrm{yr}$, where the "Hubble limit" corresponds to $\tau_{c}=10 \times 10^{9} \mathrm{yr}$. The largest of these cannot of course be actual ages. In the simplest resolution of this apparent paradox, the initial spin period of the pulsars were close to their present periods (cf. eq. 2; Camilo, Thorsett, \& Kulkarni 1994). This has been amply confirmed with optical studies of millisecond pulsar companions (Lundgren et al. 1996, also this volume), where several characteristic ages are substantially larger than white dwarf cooling ages, suggesting that accretion in mass-exchanging low-mass systems proceeds at well under Eddington-limited rates $\left(0.001 \lesssim \dot{M} / \dot{M}_{\text {Edd }} \lesssim 0.01\right)$.

Within the new framework, it is curious to notice the broad correlation between $P$ and $\dot{P}$ among those pulsars with the largest values of $\tau_{c}$ (Fig. $2 \mathrm{~b}$ ). If $\tau_{c}$ corresponded to real ages, this correlation would of course be expected, with the oldest pulsars aligned near the Hubble limit. However, a characteristic age much greater than $\sim 10^{10} \mathrm{yr}$ or than the corresponding cooling age simply suggests a low accretion rate and, a priori, one might expect to observe pulsars with $\dot{P} \lesssim 10^{-20.5}$ and $P \gtrsim 5 \mathrm{~ms}$, as one does with $P \lessgtr 5 \mathrm{~ms}$, within the limits allowed by the inferred values of $\dot{M}$. That we do not observe such pulsars may suggest that the apparent distribution of millisecond pulsars in the $P-\dot{P}$ diagram 
is determined at least in part by factors other than the original distribution in $P$ and $B$. One possibility is that we are observing a luminosity evolution for the millisecond pulsar population.

For a particular magnetic field configuration there is in principle a well established region beyond which pulsar radio emission ceases. However, as the ensemble of pulsars likely displays a variety of magnetic field arrangements, a group of pulsars with identical $B$ will cease emission over a range of $P$ (the "death valley" of Fig. 3), with the threshold for each pulsar depending in detail on the individual field topology (Chen \& Ruderman 1993). At high values of $B$ the momentum of the electron-positron pairs perpendicular to the magnetic field direction is quickly radiated away. Relaxing this condition for low $B$ values results in a "death line" largely determined by synchrotron radiation instead of curvature radiation processes. In turn the line is shifted to shorter periods compared to an extrapolation of the long period death line (Björnsson 1996, also this volume; Fig. 3). Much as for long period pulsars, the absolute location of the new limit is uncertain; more importantly, the relative position of its small- and large- $P$ sections match the observed distribution of pulsars rather impressively. As the slope of the new death line at small periods is similar to one of constant characteristic age, it is not clear whether the observed distribution of millisecond pulsars requires such a line, rather it appears consistent with one (see also Rudak \& Ritter 1994). (To determine whether a death line is in fact necessary to explain observations requires knowledge of the distribution of spin periods following accretion as a function of $B$.)

Curiously, the two most luminous millisecond pulsars (PSRs B1937+21 and J0218+4232) are those furthest from the death line of Figure 3. They are presumably young objects, with $\tau_{c}<5 \times 10^{8} \mathrm{yr}$, but youth alone does not imply high radio luminosity, as PSR J1012+5307, very young and of median luminosity (Lorimer et al. 1995), suggests. On the other hand there is some debate regarding the age of this pulsar (Alberts et al. 1996). It is clearly important that believable cooling models for $m_{2} \lesssim 0.3 \mathrm{M}_{\odot}$ white dwarfs be developed.

Some basic issues concerning the recycling model remain unresolved. About $20 \%$ of all disk millisecond pulsars are isolated and many of them, with little spindown energy, are unlikely to have ablated their companions, so that their origin remains unclear. According to the recycling model, as much as $\sim 1 \mathrm{M}_{\odot}$ may be transferred to the compact object in the course of conservative Roche-lobe overflow evolution of some tight binary systems (only $\sim 0.1 \mathrm{M}_{\odot}$ is required to spin neutron stars up to $\sim 1.5 \mathrm{~ms}$ and, if instabilities develop in the mass transfer process, the balance can be blown off by a millisecond pulsar reborn near the spin-up line (Ruderman, Shaham, \& Tavani 1989), but, given the mass transfer rates inferred from optical studies of white dwarf-millisecond pulsar binaries, it is unclear whether young millisecond pulsars are powerful enough to do that); thus one may expect to find $m_{1} \gtrsim 2 \mathrm{M}_{\odot}$ neutron stars. - Future observational work, e.g., in the areas of further millisecond pulsar surveys, proper motion measurements, optical studies of white dwarf companions, and mass determination by "Shapiro delay" timing measurements, will have much to add concerning the validity of the recycling model, and therefore evolution of magnetic fields. 
Acknowledgments. I thank Dipankar Bhattacharya and Andrew Lyne for enlightening discussions, and gratefully acknowledge a fellowship under the auspices of the European Commission.

\section{References}

Alberts, F., Savonije, G. J., Pols, O. R., \& van den Heuvel, E. P. J. 1996, Nature. In press

Bhattacharya, D. \& van den Heuvel, E. P. J. 1991, Phys. Rep., 203, 1

Bhattacharya, D., Wijers, R. A. M. J., Hartman, J. W., \& Verbunt, F. 1992, A\&A, 254, 198

Björnsson, C.-I. 1996, ApJ. In press

Blandford, R. D. \& Romani, R. W. 1988, MNRAS, 234, 57P

Boyd, P. T. et al. 1995, ApJ, 448, 365

Camilo, F., Thorsett, S. E., \& Kulkarni, S. R. 1994, ApJ, 421, L15

Chen, K. \& Ruderman, M. 1993, ApJ, 402, 264

Deshpande, A. A., Ramachandran, R., \& Srinivasan, G. 1995, J. Astrophys. Astr., 16, 69

Geppert, U. \& Urpin, V. 1994, MNRAS, 271, 490

Gunn, J. E. \& Ostriker, J. P. 1970, ApJ, 160, 979

Harrison, P. A., Lyne, A. G., \& Anderson, B. 1993, MNRAS, 261, 113

Jahan Miri, M. \& Bhattacharya, D. 1994, MNRAS, 269, 455

Kaspi, V. M., Manchester, R. N., Siegman, B., Johnston, S., \& Lyne, A. G. 1994, ApJ, 422, L83

Kulkarni, S. R. 1986, ApJ, 306, L85

Kulkarni, S. R. \& Anderson, S. B. 1996, in Dynamical Evolution of Star Clusters - Confrontation of Theory and Observations: IAU Symposium 174, available at http://astro.caltech.edu/ srk/gciau.ps

Lorimer, D. R., Festin, L., Lyne, A. G., \& Nicastro, L. 1995, Nature, 376, 393

Lundgren, S. C., Cordes, J. M., Foster, R. S., Wolszczan, A., \& Camilo, F. 1996, ApJ, 458, L33

Lyne, A. G., Anderson, B., \& Salter, M. J. 1982, MNRAS, 201, 503

Lyne, A. G. \& Lorimer, D. R. 1994, Nature, 369, 127

Lyne, A. G., Pritchard, R. S., \& Smith, F. G. 1993, MNRAS, 265, 1003

Lyne, A. G., Pritchard, R. S., Smith, F. G., \& Camilo, F. 1996, Nature. In press

Manchester, R. N. \& Taylor, J. H. 1977, Pulsars, (San Francisco: Freeman)

Narayan, R. \& Ostriker, J. P. 1990, ApJ, 352, 222

Romani, R. W. 1990, Nature, 347, 741

Rudak, B. \& Ritter, H. 1994, MNRAS, 267, 513

Ruderman, M., Shaham, J., \& Tavani, M. 1989, ApJ, 336, 50

Urpin, V. \& Geppert, U. 1995, MNRAS, 275, 1117

Urpin, V. A. \& Muslimov, A. G. 1992, MNRAS, 256, 261

Wakatsuki, S., Hikita, A., Sato, N., \& Itoh, N. 1992, ApJ, 392, 628

Young, E. J. \& Chanmugam, G. 1995, ApJ, 442, L53 